\title{
Domination Numbers of Trees
}

\author{
Min-Jen Jou* and Jenq-Jong Lin \\ Ling Tung University, Taichung 40852, Taiwan \\ ${ }^{*}$ Corresponding author
}

\begin{abstract}
A set $S$ of vertices is a dominating set of $G$ if $N_{G}[S]=V(G)$. The domination number $\gamma(G)$ of a graph $G$ is the minimum cardinality among all dominating sets of $G$. The decision problem of determining the domination number for arbitrary graphs is NP-complete. Here we focus on trees. If $x$ and $x^{\prime}$ are duplicated leaves adjacent to the same support vertex in a tree $T$, then $\gamma\left(T-x^{\prime}\right)=\gamma(T)$. If $T^{\prime}$ can be obtained from $T$ by adding some duplicated leaves, we can see that $\gamma\left(T^{\prime}\right)=\gamma(T)$. So the maximum order of a tree $T$, which is $\gamma(T)=k$, is infinity. In this paper, we focus on trees which are without duplicated leaves. For $k \geqq 1$, we determine the minimum and maximum orders of the trees $T$ which are without duplicated leaves and $\gamma(T)=k$. Moreover, we characterize the trees of minimum and maximum orders.
\end{abstract}

Keywords- domination number; tree; order; duplicated leaves

\section{INTRODUCTION}

All graphs considered in this paper are finite, loopless, and without multiple edges. For a graph $G, V(G)$ and $E(G)$ denote the vertex set and the edge set of $G$, respectively. The cardinality of $V(G)$ is called the order of $G$, denoted by $|G|$. The (open ) neighborhood $N_{G}(v)$ of a vertex $v$ is the set of vertices adjacent to $v$ in $G$, and the close neighborhood $N_{\mathrm{G}}[v]$ is $N[v]=N(v) \cup\{v\}$. For any subset $A \subseteq V(G)$, denote $N(A)=\bigcup_{v \in A} N(v)$ and $N[A]=\bigcup_{x \in A} N[v]$. The degree of $v$ is the cardinality of $N_{G}(v)$, denoted by $\operatorname{deg}_{G}(v)$. Two distinct vertices $u$ and $v$ are called duplicated in $G$ if $N_{G}(u)=N_{G}(v)$. A vertex $x$ is said to be leaf if $\operatorname{deg}_{G}(v)=1$. A vertex of $G$ is a suppor vertex if it is adjacent to a leaf in $G$. We denote by $L(G)$ and $U(G)$ the collections of all leaves and support vertices of $G$, respectively. For a subset $A \subseteq V(G)$, the deletion of $A$ from $G$ is the graph $G-A$ obtained by removing all vertices in $A$ and all edges incident to these vertices. The union of two disjoint graphs $G_{1}$ and $G_{2}$ is the graph $G_{1} \cup G_{2}$ with vertex set $\mathrm{V}\left(G_{1} \cup G_{2}\right)=\mathrm{V}\left(G_{1}\right) \cup$ $\mathrm{V}\left(G_{2}\right)$ and edge set $\mathrm{E}\left(G_{1} \cup G_{2}\right)=\mathrm{E}\left(G_{1}\right) \cup \mathrm{E}\left(G_{2}\right)$. A forest is a graph with no cycles, and a tree is a connected forest. If $u$ and $v$ are duplicated vertices in a tree, then they are both leaves. The $n$-path $P_{n}$ is a path of order $n$. For other undefined notions, the reader is referred to [1] for graph theory.

A set $S$ of vertices is a dominating set of $G$ if $N_{G}[S]=V(G)$. The domination number $\gamma(G)$ of $G$ is defined to be the minimum cardinality among all dominating sets of $G$. A dominating set of cardinality $\gamma(G)$ in $G$ is said to be a $\gamma$-set. A $\gamma$-set containing all support vertices of $G$ is called a $\gamma_{U}$-set. One of the fastest growing areas within graph theory is the study of domination and related subset problems. A dominating set have been proposed as a virtual backbone for routing in wireless ad hoc networks (see [6]). The topology of such wireless ad hoc network can be modeled as a unit-disk graph (UDG), a geometric graph in which there is an edge between two vertices if and only if their distance is at most one. A dominating set of a wireless ad hoc network is a dominating set of the corresponding UDG. The discussion of domination in graphs are initiated by Ore [5]. Several decades later, domination and its variations in graphs are well studied, an estimated thousand papers have been written on this topic (see [2],[3],[4]).

The decision problem of determining the domination number for arbitrary graphs is NP-complete. Here we focus on trees. If $x$ and $x^{\prime}$ are duplicated leaves adjacent to the same support vertex in a tree $T$, then $\gamma\left(T-x^{\prime}\right)=\gamma(T)$. If $T^{\prime}$ can be obtained from $T$ by adding some duplicated leaves, we can see that $\gamma\left(T^{\prime}\right)=\gamma(T)$. So the maximum order of a tree $T$, which is $\gamma(T)=k$, is infinity. In this paper, we focus on trees which are without duplicated leaves. For $k \geq 1$, we determine the minimum and maximum orders of the trees $T$ which are without duplicated leaves and $\gamma(T)=k$. Moreover, we characterize the trees of minimum and maximum orders.

\section{PRELIMINARY}

We need the following lemmas.

Lemma 2.1. If $u v$ is an edge of a connected graph $G$ and $G-u v=G_{1} \cup G_{2}$, then $\gamma(G) \leqslant \gamma\left(G_{1}\right)+\gamma\left(G_{2}\right)$.

Proof. Suppose $u v$ is an edge of a connected graph $G$ and $G-u v=G_{1} \cup G_{2}$. Let $S$ i be a $\gamma$-set of $G_{i}$ for $i=1$ and 2 . Suppose $S=S_{1} \cup S_{2}$, then $N[S]=N\left[S_{1} \cup S_{2}\right]=N\left[S_{1}\right] \cup N\left[S_{2}\right]=V(G)$. So $S$ is a dominating of $G$, thus $\gamma(G) \leq|S|=\left|S_{1}\right|+\left|S_{2}\right|$ $=\gamma\left(G_{1}\right)+\gamma\left(G_{2}\right)$.

Lemma 2.2. If $G$ is a graph with at least three vertices, then there exists a $\gamma_{U}$-set of $G$.

Proof. Suppose $G$ is a graph with at least three vertices and let $S$ be a $\gamma$-set $S$ of $G$. If $S$ is a $\gamma_{U}$-set of $G$, then we are done. So we assume that $A=U(T)-S \neq \phi$, and let $B=L(T) \cap N(A)$. Then $B \subseteq S$ and $|B| \geq|A|$. Let $S^{\prime}=(S-B) \cup A$. Then $N\left[S^{\prime}\right]=V(G)$, so $S^{\prime}$ is a dominating set of $G$. Thus $|S|=\gamma(G) \leq\left|S^{\prime}\right|=|S|-|B|+|A| \leq|S|$, the equalities hold and $S^{\prime}$ is a $\gamma_{U}$-set of $G$.

Lemma 2.3. If $x$ and $x^{\prime}$ are two duplicated leaves adjacent to 
the same support vertex in a graph $G$, then $\gamma\left(G-x^{\prime}\right)=\gamma(G)$.

Proof. Suppose $x$ and $x^{\prime}$ are two duplicated leaves adjacent to the same support vertex in a graph $G$, and let $G^{\prime}=G-x$. If $S$ is a $\gamma_{U}$-set of $G$, then $S$ is a $\gamma_{U}$-set of $G^{\prime}$. So $\gamma\left(G-x^{\prime}\right)=$ $\gamma\left(G^{\prime}\right)=|S|=\gamma(G)$.

\section{MAIN THEOREM}

By Lemma 2.3, we can see that the maximum order of a tree $T$, which is $\gamma(T)=k$, is infinity. Thus we focus on trees which are without duplicated leaves. First, we determine the minimum order of the trees $T$ which are without duplicated leaves and $\gamma(T)=k$. Moreover, we characterize the trees of the minimum order.

Theorem 3.1. If $T$ is a tree with at least two vertices and $\gamma(T)=k$, where $k \geq 1$, then $|T| \geq 2 k$.

Proof. Suppose $T$ is a tree with at least two vertices and $\gamma(T)=k$, where $k \geq 1$. Let $S$ be a $\gamma$-set of $T$. Then $N[S]=V(G)=N[V(T)-S]$, so $S^{c}=V(T)-S$ is a dominating set of $T$. Hence $\left|S^{c}\right| \geq k$ and $|T|=|S|+\left|S^{c}\right| \geq k+k=2 k$.

Lemma 3.2. Let $T$ be a tree with at least two vertices and $\gamma(T)=k$, where $k \geq 1$. If $|T|=2 k$, then $T$ has no duplicated leaf.

Proof. Let $T$ be a tree of order $2 k$ and $\gamma(T)=k$, where $k \geq 1$. Suppose that there exist two distinct leaves $x$ and $x^{\prime}$ adjacent to $y$ in $T$, by Lemma 2.3, then $\gamma\left(T-x^{\prime}\right)=\gamma(T)=k$. Note that $T^{\prime}=T-x^{\prime}$ is a tree. By Theorem 3.1, $\left|T^{\prime}\right| \geq 2 k$. Thus $|T|=\left|T^{\prime}\right|+1 \geq 2 k+1$. This is a contradiction, we complete the proof.

Theorem 3.3. Let $T$ be a tree with at least two vertices and $\gamma(T)=k$, where $k \geq 1$. If $|T|=2 k$, then $V(T)=U(T) \bigcup L(T)$ and $|U(T)|=k$.

Proof. We prove this theorem by induction on $k \geq 1$. If $k=1$, then $T=P_{2}$. If $k=2$, then $T=P_{4}$. It's true for $k=1$ and 2. Let $k \geq 3$. Assume that it's true for all $k^{\prime}<k$. Suppose that $T$ is a tree of order $2 k$ and $\gamma(T)=k$. Let $P_{i}: x_{i}, y_{i}, z, w, u, \ldots$ be a longest path of $T$, where $\left|P_{i}\right|=m \geq 5$ and $i=1, \ldots, a$. By Lemma 3.2, then $\left|N\left(y_{i}\right) \bigcap L(T)\right|=1$ for every $i$. Let $A=\left\{y_{1}, \ldots, y_{a}\right\}$. If $m=5$, then $a=k-2$. Thus $z \in U(T)$ and $U(T)=A \bigcup\{z, w\}$. So it's true for $m=5$. Thus we assume that $m \geq 6$.

Claim 1. $z \in U(T)$. Suppose that $z \notin U(T)$, then $N(z)=A \bigcup\{w\}$ and $H=T-N[A]$ is tree of order $|H|=|T|-(2 a+1)=2(k-a)-1 \geq 3$. By Theorem 3.1, $\gamma(H) \leq k-a-1$. Note that $z \in N(A)$. By Lemma 2.1, $k=\gamma(T) \leq|A|+\gamma(H) \leq a+(k-a-1)=k-1$. This is a contradiction, so $z \in U(T)$.
Let $z^{\prime}$ be the leaf of $z$ in $T$ and $T^{\prime}=T-\left(N[A] \cup\left\{z, z^{\prime}\right\}\right)$ and $T^{*}=T-V\left(T^{\prime}\right)$. Then $T$ is a tree of order $\left|T^{\prime}\right|=|T|-(2 a+2)=2(k-a-1) \geq 3$. By Theorem 3.1, $\gamma\left(T^{\prime}\right) \leq k-a-1$. Note that $z w$ is an edge of $T$ such that

$T-Z w=T^{*} \cup T \quad$ by $\quad$ Lemma 2.1, $k=\gamma(T) \leq \gamma\left(T^{*}\right)+\gamma\left(T^{\prime}\right) \leq \quad(a+1)+(k-a-1)=k . \quad$ The equalities hold, $\gamma\left(T^{\prime}\right)=k-a-1$. Hence $T$ is a tree of order $\left|T^{\prime}\right|=2(k-a-1) \quad$ and $\quad \gamma\left(T^{\prime}\right)=k-a-1 \quad$, by induction hypothesis,

$V\left(T^{\prime}\right)=U\left(T^{\prime}\right) \bigcup L\left(T^{\prime}\right)$.

Claim 2. $w \in U\left(T^{\prime}\right)$. Suppose that $w \notin U\left(T^{\prime}\right)$, then $w \in L\left(T^{\prime}\right)$ and $T^{\prime \prime}=T^{\prime}-\{w\}$ is a tree of order $\left|T^{\prime \prime}\right|=\left|T^{\prime}\right|-1=2(k-a-1)-1 \geq 2$. Hence, by Theorem 3.1, we have that $\gamma\left(T^{\prime \prime}\right) \leq k-a-2$. Note that $w \in N(z)$ and $T-w u=\left(T-V\left(T^{\prime \prime}\right)\right) \cup T^{\prime \prime}$. By Lemma 2.1, $k=\gamma(T) \leq \gamma\left(T-V\left(T^{\prime \prime}\right)\right)+\gamma\left(T^{\prime \prime}\right) \leq(a+1)+(k-a-2)=k-1$. This is a contradiction, so $w \in U\left(T^{\prime}\right)$.

By Claim 2, we can see that $U(T)=A \bigcup\{z\} \bigcup U\left(T^{\prime}\right)$. That is $V(T)=U(T) \bigcup L(T)$ and $|U(T)|=k$. Hence it's true for $k$, we complete the proof.

Now we determine the maximum order of the trees $T$ which are without duplicated leaves and $\gamma(T)=k$. Moreover, we characterize the trees of the maximum orders. Let $\Omega(k)$ be the collection of trees $T$ which hold the following properties.

(i) $T$ has no duplicated leaf.

(ii) $\gamma(T)=|U(T)|=k$.

(iii)For each $v \in U(T), \delta(v)=\min \{d(u, v): u \in U(T)\}=3$, where $d(u, v)$ is the distance between $u$ and $v$.

Lemma 3.4. Suppose $T \in \Omega(k)$, then $T$ is a tree without duplicated leaves of order $|T|=4 k-2$ and $U(T)$ is a $\gamma_{U}$-set of $T$, where $\gamma(T)=|U(T)|=k$.

Theorem 3.5. Suppose $T$ is a tree without duplicated leaves and $\gamma(T)=k$, where $k \geq 1$. Then $|T| \leq 4 k-2$. The equality holds if and only if $T \in \Omega(k)$.

Proof. It's true for $k=1$, so we assume that $k \geq 2$. Suppose that $T$ is a tree without duplicated leaves and $\gamma(T)=k$ such that $|T|$ is as large as possible. By Lemma 3.4, then we obtain that $|T| \geq 4 k-2$. Let $S$ be a $\gamma_{U}$-set of $T$. Since $|T|$ is as large as possible, we obtain that $S=U(T)$ and $N[u] \cap N[v]=\phi$ for $u \neq v$ in $S$. Thus $\mid V(T)-(U(T) \cup L(T) \mid \leq 2(|S|-1)=2 k-2$. Hence $\quad 4 k-2 \leq|T|=|U(T) \cup L(T)|+|V(T)-(U(T) \cup L(T))| \leq$ $2 k+(2 k-2)=4 k-2$. The equalities hold, $\gamma(T)=|U(T)|=$ 
$|L(T)|=k \quad$ and $\quad \mid V(T)-(U(T) \bigcup L(T) \mid=2(|S|-1)=2 k-2$.

That is $T \in \Omega(k)$.

\section{REFERENCES}

[1] J.A. Bondy, USR Murty and Graph Theory with Application, New York, 1976.

[2] T.W. Haynes, S.T. Hedetniemi and P.J. Slater, Fundamentals of domination in graphs, Marcel Dekker Inc., New York, 1998.

[3] T.W. Haynes, S.T. Hedetniemi and P.J. Slater, Fundamentals of domination in graphs:Advanced Topics, Marcel Dekker Inc., New York, 1998.

[4] M.J. Jou, "Dominating sets and independent sets in a tree," to appear in Ars Combinatoria.

[5] O. Ore, Theory of graphs, in: Amer. Math. Soc. Colleq., Vol. 38, Providence, RI, 1962.

[6] Peng-Jun Wan, Khaled M. Alzoubi and Ophir Frieder, "Distributed Construction of Connected Dominating Set in Wireless Ad Hoc Networks," Mobile Networks and Applications, Vol. 9, Issue 2, pp 141149, April 2004. 